\title{
Low reporting of adverse events following immunization, during a Measles-Rubella catchup and Polio mass vaccination campaign in Uganda; 2019
}

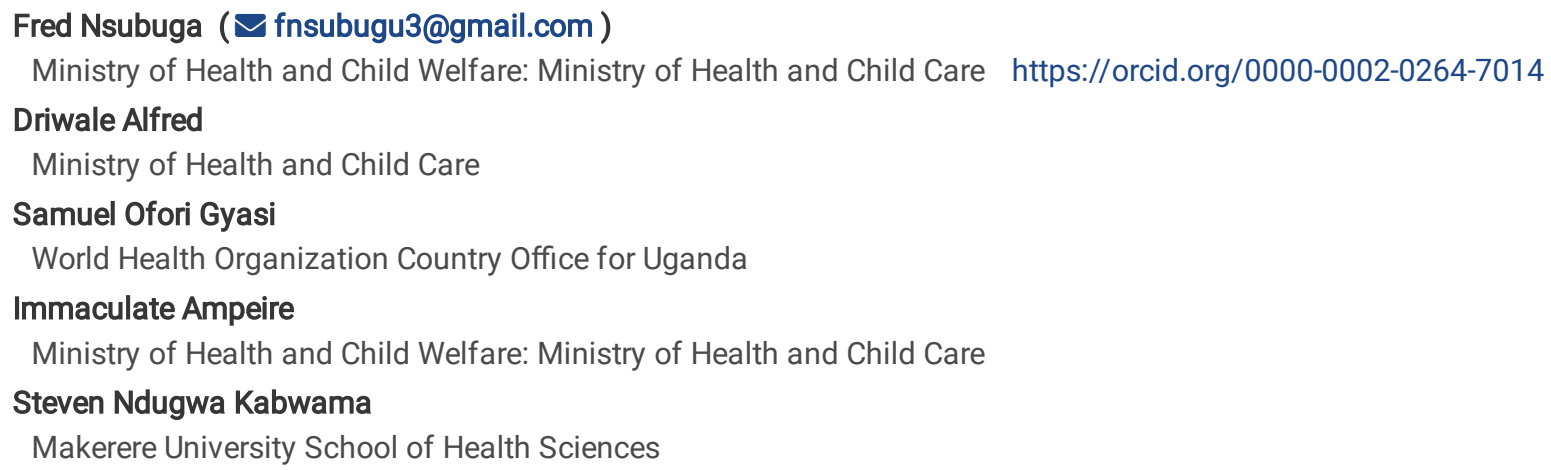

Research

Keywords: Adverse event following immunization, Uganda, Measles-Rubella catch-up campaign

Posted Date: January 26th, 2022

DOI: https://doi.org/10.21203/rs.3.rs-1286703/v1

License: (c) (i) This work is licensed under a Creative Commons Attribution 4.0 International License. Read Full License 


\section{Abstract \\ Background}

Vaccine safety surveillance is increasingly becoming a critical area in national immunisation programs. Uganda conducted a Measles-Rubella catch-up and Polio mass vaccination campaign between 16 and 20 October 2019. We conducted a descriptive analysis of the adverse events following immunisation reported following this campaign to inform policy updates. The target age-group for the campaign was children aged 9 months to 14 completed years, while the target age-group for polio campaign was 0-59 months. We conducted active search for the serious adverse event following immunisation that were reported.

\section{Results}

Among the 19,241,348 persons vaccinated during the Measles-Rubella catch-up and Polio mass campaign, 10,612,278 (55\%) received MR vaccine alone; while $8,629,070$ (45\%) received either bivalent oral polio vaccine (bOPV) alone or MR and bOPV for children 9 to 59 months. The mean age of the children was 8.3 years, range $0.5-14$ years, including 68 (47\%) boys, 64 (44\%) girls and 13 (9\%) missing sex classification. The serious adverse events following immunisation (AEFI) included; toxic epidermal necrolysis 3 (2.1\%), Steven Johnson syndrome 2 (1.4\%), severe anemia 2 (1.4\%), febrile illness 1 (0.7\%), sudden respiratory failure $1(0.7 \%)$, bullous impetigo $1(0.7 \%)$ and injection abscess 19 (13\%).

\section{Conclusion}

AEFI reporting in Uganda is low, and concerted efforts need to be undertaken to create community awareness about the importance of reporting. Additionally, the health care systems should be strengthened to efficiently investigate all potential signals that led to quality causality assessment of serious and other AEFI of public health concern so as to provide correct information to the community and prevent false association of vaccines and or vaccination to incorrect signals, and build a resilient immunisation program that is trusted by the community.

\section{Background}

Immunisation is known to be among the most cost-effective public health interventions globally (1). Nevertheless, like all other pharmaceutical products, vaccines are not entirely risk-free. Following vaccination, vaccine recipients often have a proclivity for experiencing adverse events following immunisation (AEFI). An AEFI is any untoward medical occurrence which follows immunization and which does not necessarily have a causal relationship with the usage of the vaccine. The adverse event may be any unfavorable or unintended sign, abnormal laboratory finding, symptom or disease (2). Furthermore, since parents bring their children for vaccination when they are healthy, their tolerance for vaccine risks is understandably lower than the tolerance for risks from drugs administered to ill persons (3).Therefore, vaccine safety has been identified as an area that needs great attention if immunisation programs are to be strengthened $(4,5)$. In line with the global and regional plans of improved vaccine pharmacovigilance, Uganda started participating in the global network for post-marketing surveillance of vaccines in 2008 and established a national adverse event following immunisation (AEFI) committee in 2012. Since then, Uganda has introduced 6 new vaccines into its routine vaccination schedule.

Parents and caretakers have been known to shun vaccination for fear of vaccine reactions, some of which may be real or perceived (6). It is therefore important to promptly respond to all vaccine safety signals to identify all rare events from which true adverse events could occur and preserve the integrity of the immunisation program. Additionally, information about the extent of AEFI can inform management strategies to increase vaccine uptake and confidence in immunization programs.

The objective of our study was to epidemiologically characterize the AEFls reported following the Measles-Rubella catch-up and Polio mass vaccination campaign to inform policy updates.

\section{Methods}

We conducted a descriptive analysis of data from the Measles-Rubella (MR) catch-up and Polio mass vaccination campaign that was implemented between 16 and 20 October 2019. The target age-group for the MR campaign was children aged 9 months to 14 completed years (i.e., 9 months to less than 15 years) while the target age-group for polio campaign was 0-59 months. Prior to the implementation of the campaign, 2 readiness assessments were conducted in all the 14 regions of the country to assess the level of preparedness of the districts to conduct the campaign. The regional assessors used a national readiness assessment tool to capture data under 4 strategic areas; (a) planning, coordination and funding, (b) monitoring and supervision, (c) vaccine, cold chain and logistics, (d) social mobilization and risk communication. The first assessment was in August 2019, while the second was conducted in September 2019. During these assessments, districts were 
supported to prepare for the detection, reporting, assessment, investigation and management of AEFI. There were also several trainings that were conducted at central, regional, district and sub-county levels to prepare the supervisors and health workers to detect and respond to AEFI.

For the first three days of the campaign, vaccinations were administered to children at schools where approximately $80 \%$ of eligible children for MR were expected to be found. The latter two days targeted approximately $20 \%$ of children who were not in schools and vaccinated at health facilities (both public and private) and other community sites designated by the district health offices. However, at the end of the official 5-day period, there were still high numbers of children who had not yet received the MR and polio vaccines. This unprecedented high number of children who were yearning to be vaccinated prompted the Minister of Health to extend the campaign for 2 days in the metropolitan Kampala (Kampala, Mukono and Wakiso) including Karamoja region; and one day for the rest of the country.

We conducted surveillance of AEFI during the MR catch-up campaign through the District Health Information Software 2 (DHIS2), which is the official ministry of health surveillance and reporting system that reports aggregate data (7). We also conducted active search for AEFI that were reported by care takers and parents of the vaccinated children, media, the teachers of schools where vaccination was conducted. We defined an $\mathrm{AEFI}$ as serious if it resulted in death, was life threatening, required in-patient hospitalization or prolongation of existing hospitalization, resulted in persistent or significant disability/incapacity, or was a congenital anomaly/birth defect (8). The serious AEFI case reports were then selected and submitted to the AEFI review committee for causality assessment. All the non-serious AEFIs were managed at the vaccination site by the health workers, while the serious AEFI cases were admitted at the nearest hospital for proper investigation and management. Data analysis was done using excel 2010 and Epi-Info 7.1.

\section{Results}

A total of 19,241,348 children were vaccinated during the Measles-Rubella catch-up and Polio mass campaign, including 10,612,278 (55\%) who received MR vaccine alone; 8,629,070 (45\%) who received either bivalent oral polio vaccine (bOPV) alone for children aged less than 9 months or MR and bOPV for children 9 to 59 months. Of the 19,241,348 doses of MR vaccine administered, we received 145 reports of AEFI that occurred within 30 days of administration of the vaccine ( 0.8 per 100,000 doses). Of the $145 \mathrm{AEFI}$ reports received, 43 ( 0.2 per 100,000 doses) were serious, while the rest 102 (0.5 per 100,000 doses) were non-serious AEFI reports (Fig. 1).

\section{Fig. 1: Distribution of AEFI cases during MR catch-up campaign: October 2019}

\section{Legend}

The rate of case-based serious AEFI reported during the campaign was 2.2 per 1000,000 doses. The mean age of the case-patients was 8.3 years, range $0.5-14$ years, with the majority 68 (47\%) boys, 64 (44\%) girls and $13(9 \%)$ missing sex classification. The serious AEFls included; toxic epidermal necrolysis 3 (2.1\%), Steven Johnson syndrome (SJS) 2 (1.4\%), severe anemia 2 (1.4\%), febrile illness1 (0.7\%), sudden respiratory failure $1(0.7 \%)$, bullous impetigo 1 ( $0.7 \%)$ and injection abscess $19(13 \%) .18 / 19$ (95\%) of the injection abscess were from one district local government. Some of the non-serious AEFIs reported included; arthralgia 2 (1.4\%), urticaria 81 (56\%), fever 3 (2.1\%), mumps 3 (2.1\%) (Fig. 2).

\section{Fig. 2: Provisional diagnoses of the different AEFI during the Oct 2019 MR catch-up campaign}

Among the serious events, 6 (14\%) died; these included toxic epidermal necrolysis 1, severe anemia 2, severe pneumonia 1, sudden respiratory failure 1 and unexplained sudden death 1 . The Western region submitted the least number of AEFI reports (Table 1). Although all regions reported, $90 / 128(70 \%)$ of the districts did not report any $\mathrm{AEFI}$.

Table 1: Distribution of AEFI by Region during the MR-Polio campaign in Uganda; Oct 2019

\begin{tabular}{|llll|}
\hline Region & Number vaccinated & Number of AEFI & Rate/100,000 \\
\hline Central & 5225389 & 53 & 1.0 \\
\hline Eastern & 5007700 & 50 & 1.0 \\
\hline North & 4047537 & 36 & 0.9 \\
\hline Western & 4960722 & 6 & 0.1 \\
\hline Total & $\mathbf{1 , 9 2 4 1 , 3 4 8}$ & 145 & 0.8 \\
\hline
\end{tabular}


Causality assessment was done for $10 / 43$ (23\%) of the serious AEFI cases with clinical details available. Of these $2 / 10$ (20\%) were classified as consistent with causal association to immunisation (sudden respiratory failure, and injection abscess), 40\% (4/10) were indeterminate (Toxic Epidermal Necrolysis 2, and Steven Johnson Syndrome 2) and 40\% (4/10) were coincidental (Toxic Epidermal Necrolysis 1, severe anemia 1, severe pneumonia 1, and bullous impetigo 1). The distribution of the AEFI cases by level of seriousness is shown in (Table 2).

Table 2

Distribution of $\mathrm{AEFI}$ case patients by age-group and seriousness, during the Oct 2019 MR catch-up campaign

\begin{tabular}{|llllll|}
\hline Age-group (years) & Frequency* & \multicolumn{5}{l|}{ Type of reaction } \\
\hline & & Non-serious & \multicolumn{2}{l|}{ Serious } \\
\hline $\mathbf{0 . 5 - 0 . 9 2}$ & & $\mathrm{n}$ & $\%$ & $\mathrm{n}$ & $\%$ \\
\hline $1-4$ & 7 & 4 & 3.96 & 3 & 7.32 \\
\hline $\mathbf{5}-\mathbf{9}$ & 37 & 25 & 24.75 & 12 & 29.27 \\
\hline $\mathbf{1 0 - 1 4}$ & 31 & 18 & 17.82 & 13 & 31.71 \\
\hline $\mathbf{0 . 5 - 1 4}$ & 67 & 54 & 53.47 & 13 & 31.71 \\
\hline
\end{tabular}

\section{*3 lacked age categorisation}

\section{Discussion}

Our analysis of the AEFI data of the Measles-Rubella catch-up and Polio mass vaccination campaign shows that all the four regions of Uganda were able to report AEFI cases. However, at sub-national level two thirds of the districts did not report any AEFI. Slightly more than threequarters of the serious AEFIs that had causality assessment done were either indeterminate or were classified as inconsistent with causal association to immunisation. Additionally, almost all the injection abscesses were reported from one district.

The Global Advisory Committee on Vaccine Safety (GACVS) has defined a benchmark for determining the functionality of a country's AEFI surveillance system as a ratio of $10 \mathrm{AEFI}$ reports per 100,000 surviving infants per year. Furthermore, with the introduction of the immunisation agenda 2030 (IA2030), another indicator which monitors the progress of AEFI surveillance in all age groups has been suggested to be one serious case report per 1,000,000 individuals per year (9). Findings from our study show that the rate of case-based serious AEFIs reported during the campaign was 2.2 per 1,000,000 individuals. This rate is slightly higher than the GACVS initial target of 1 serious AEFI case report per 1,000,000 population per year. However, this rate could be an artifact of heightened surveillance as it was achieved during a campaign setting where deliberate efforts were undertaken to train and sensitize health workers and communities before and during the campaign to ensure prompt detection, reporting and investigation of serious AEFI. Also, AEFI regional teams were trained to support district teams in their area of operation to conduct investigation of all serious AEFI. Although this reporting indicator was achieved at the national level, $70 \%$ of the districts did not report any AEFI. Failure to report may imply that the surveillance system of a District Local Government is not prepared to detect and respond to AEFI or other safety signals that may be of public concern. Its critical therefore to strengthen AEFI detection, reporting and investigation of serious AEFI at the sub-national level, if we are to achieve the global target of 10 serious AEFI cases per $1,000,000$ population per year by 2030 . Studies done elsewhere have showed that AEFI reporting is a key ingredient in monitoring vaccine safety and improving public confidence in vaccines $(10,11)$.

Our analysis indicated that the Central and Eastern regions reported the highest rates ( 1 per 100,000), while the Western region reported the least (0.1 per 100,000). Overall the reporting rate was low (0.8 per 100 000) compared to Australia 5 per 100,000 (12). Lower rates of AEFI reporting from districts have been noted from other countries. A study done in Nigeria showed that $19 \%$ of the states that participated in the 2017/18 Measles vaccination campaign had no AEFI report (13). This is in stark contrast to a study done in Zhejiang Province, China which showed higher AEFI reporting rates across all municipalities ranging from 30.5 per 100,000 in Quzhou to the lowest reporting Huzhou at 3.0 per 100,000 doses (14).

Other countries that have reported lower rates include India in July 2009 following a mass vaccination campaign against measles that was conducted in West Bengal (15).

The MR vaccine used by the national immunisation programs is safe and efficacious and has been recommended by the World Health Organisation (WHO) to be used globally $(16,17)$. Uganda undertook this unprecedented catchup campaign to introduce the Rubella vaccine into the national immunisation program in addition to the control the measles-rubella outbreaks that had occurred since 2018 . Following this campaign, the causality committee conducted an assessment for 10/43 (23\%) of the serious AEFI cases with clinical details available. Of 
these, $20 \%$ were classified as consistent with causal association to immunisation (sudden respiratory failure -A3, and injection abscess-A3), 40\% were indeterminate (Toxic Epidermal Necrolysis 2, and Steven Johnson Syndrome 2) and 40\% were coincidental (Toxic Epidermal Necrolysis 1, severe anemia 1, severe pneumonia 1, and bullous impetigo 1). An indeterminate classification is where the event followed vaccination (temporal relationship) but there is inadequate definitive evidence for vaccine causing the event. Additionally, inconsistent causal association to immunisation means the underlying condition is caused by exposure to something other than the vaccine (coincidental). It is therefore important to conduct causality assessment of AEFI to help immunisation programs to make evidence based decisions and to respond effectively to vaccine safety concerns $(18,19)$. The sudden respiratory failure was categorized as an immunisation error (A3).Our findings contrasts those found in the Nigerian study which found out that $50 \%$ of the serious AEFls were vaccine product related reactionsA1(13). Another study conducted in Georgia in 2008 found that $63.3 \%$ of the AEFI perceived as serious following measles-rubella immunisation campaign were likely related to vaccination(20).

One cluster of AEFI which involved 18 persons with injection abscesses was reported from one of the districts in northern Uganda. Injection abscesses are usually categorized as immunisation error related reaction(21). These errors usually result from vaccine storage, transportation, handling, preparation, and administration. Immunisation programs need to prevent them or identify them early in order to maintain trust in the program (22). A study conducted in India following a measles-rubella campaign also documented injection abscess as the second common AEFI reported(23). Studies conducted elsewhere have documented no injection abscess following MR campaign(20, 24).

\section{Conclusion}

AEFI reporting in Uganda is low, and concerted efforts need to be undertaken to create community awareness about the importance of reporting. Additionally, the health care systems should be strengthened to efficiently investigate all potential signals that led to quality causality assessment of serious and other AEFI of public health concern so as to provide correct information to the community and prevent false association of vaccines and or vaccination to incorrect signals, and build a resilient immunisation program that is trusted by the community.

\section{Abbreviations}

AEFI Adverse events following immunisation

bOPV Bivalent oral polio vaccine

DHIS2 District Health Information Software 2

GACVS Global Advisory Committee on Vaccine Safety

IA2030 Immunisation agenda 2030

MR Measles-Rubella

WHO World Health Organisation

\section{Declarations}

\section{Ethics approval and consent to participate}

The Director General of Ministry of Health approved this study because it was determined not human subjects' research. The primary purpose was to identify, characterize, and conduct descriptive epidemiology of perceived AEFIs following a public health intervention that was conducted to prevent morbidity and mortality due to an ongoing measles-rubella outbreak. Patients were referred for free treatment at Government health facilities. Verbal informed consent in the local language was sought from respondents or care-takers of diseased children. They were informed that their participation was voluntary and their refusal would not result in any negative consequences. To protect the confidentiality of the respondents, each was assigned a unique identifier which was used instead of their names.

\section{Consent for publication}

Not applicable

\section{Availability of data and materials}

The datasets generated during and/or analysed during the current study are not publicly available due to confidentiality conditions. However, they can be available upon request from the Director General Ministry of Health at Email: info@health.go.ug 


\section{Funding}

The authors were funded by their respective organizations. The funders had no role in the study design, data collection, analysis, decision to publish, or preparation of the manuscript.

\section{Authors' contributions}

FN drafted the initial manuscript. SNK, AD, FN designed and coordinated the study, FN, AD, SOG, IA, SNK performed the statistical analysis. All supervisors reviewed the initial draft and finalised the manuscript. All authors read and approved the final manuscript.

\section{Acknowledgements}

We would like to thank the National Drug Authority, Ministry of Health, World Health Organisation, District Health Team Members, and health workers who worked tirelessly to respond to all the AEFI. The team effort from all these stakeholders was extremely crucial for the success of the MR catch-up campaign.

\section{Competing interests}

The authors declare that they have no competing interests.

\section{References}

1. Walker DG, Hutubessy R, Beutels P. World Health Organisation Guide for standardisation of economic evaluations of immunization programmes. Vaccine. 2010;28(11):2356-9.

2. Gold MS, MacDonald NE, McMurtry CM, Balakrishnan MR, Heininger U, Menning L, et al. Immunization stress-related response-redefining immunization anxiety-related reaction as an adverse event following immunization. Vaccine. 2020;38(14):3015-20.

3. Bonhoeffer J, Kohl K, Chen R, Duclos P, Heijbel H, Heininger U, et al. The Brighton Collaboration: addressing the need for standardized case definitions of adverse events following immunization (AEFI). Vaccine. 2002;21(3):298-302.

4. World Health Organisation. Global vaccine safety blueprint. Department of Immunization, Vaccines and Biologicals. Available from: https://apps.who.int/iris/bitstream/handle/10665/70919/WHO_IVB_12.07_eng.pdf;jsessionid=B6DA1FB0E18F070A9E4528FD2A30F003? sequence $=1$.

5. Mesfin YM, Cheng A, Lawrie J, Buttery J. Use of routinely collected electronic healthcare data for postlicensure vaccine safety signal detection: a systematic review. BMJ Glob Health. 2019;4(4):e001065.

6. World Health Organization. Causality assessment of an adverse event following immunization (AEFI): user manual for the revised WHO classification. Available from: https://apps.who.int/iris/bitstream/handle/10665/259959/9789241513654-eng.pdf.

7. Kiberu VM, Matovu JKB, Makumbi F, Kyozira C, Mukooyo E, Wanyenze RK. Strengthening district-based health reporting through the district health management information software system: the Ugandan experience. BMC Med Inform Decis Mak vol. 2014;14:40.

8. World Health Organization. MODULE 3: Adverse events following immunization. Available from: https://www.who.int/vaccine_safety/initiative/tech_support/Part-3.pdf?ua=1.

9. World Health Organization. Vaccine safety indicator for the immunization agenda 2030. Available from: https://www.who.int/groups/global-advisory-committee-on-vaccine-safety/topics/indicator.

10. Bonhoeffer J, Black S, Izurieta H, Zuber P, Sturkenboom M. Current status and future directions of post-marketing vaccine safety monitoring with focus on USA and Europe. Biologicals. 2012;40(5):393-7.

11. Alicino C, Merlano C, Zappettini S, Schiaffino S, Della Luna G, Accardo C, et al. Routine surveillance of adverse events following immunization as an important tool to monitor vaccine safety. Hum Vaccin Immunother. 2015;11(1):91-4.

12. Turnbull FM, Burgess MA, McIntyre PB, Lambert SB, Gilbert GL, Gidding HF, et al. The Australian measles control campaign, 1998. Bull World Health Organ. 2001;79:882-8.

13. Gbenewei E, Nomhwange T, Taiwo L, Ayodeji I, Yusuf K, Jean Baptiste AE, et al. Adverse events following immunization: Findings from 2017/2018 measles vaccination campaign, Nigeria AEFI reporting in 2017/2018 measles vaccination campaign. Vaccine. 2021.

14. Hu Y, Li Q, Lin L, Chen E, Chen Y, Qi X. Surveillance for Adverse Events following Immunization from 2008 to 2011 in Zhejiang Province, China. Clin Vaccine Immunol. 2013;20(2):211-7.

15. Mallik S, Mandal PK, Ghosh P, Manna N, Chatterjee C, Chakrabarty D, et al. Mass Measles Vaccination Campaign in Aila Cyclone-Affected Areas of West Bengal, India: An In-depth Analysis and Experiences. Iran J Med Sci. 2011;36(4):300-5.

16. World Health Organization. Measles vaccines: WHO position paper, April 2017-Recommendations. Vaccine. 2019, 37(2):219-222. 
17. World Health Organization. Causality assessment of adverse events following immunization. Weekly Epidemiological Record= Relevé épidémiologique hebdomadaire. 2001;76(12):85-9.

18. Stefanizzi P, Calabrese G, Infantino V, Del Matto G, Tafuri S, Quarto M. SYSTEMATIC USE OF CAUSALITY ASSESSMENT IN AEFI SURVEILLANCE: A 2013-2016 PILOT STUDY IN PUGLIA. EuroMediterranean Biomed. J. 2017, 12.

19. Khetsuriani N, Imnadze P, Baidoshvili L, Jabidze L, Tatishili N, Kurtsikashvili G, et al. Impact of unfounded vaccine safety concerns on the nationwide measles-rubella immunization campaign, Georgia, 2008. Vaccine. 2010, 28(39):6455-6462.

20. World Health Organisation. WHO Vaccine Safety Basics. Available from: https://vaccine-safety-training.org/immunization-error-relatedreaction.html.

21. World Health Organization. Global manual on surveillance of adverse events following immunization. 2014. Available from: https://apps.who.int/iris/handle/10665/206144.

22. Rajkumari B, Keisam A, Haobam D, Thounaojam T. Evaluation of vaccination coverage of measles-rubella campaign in Imphal East District, Manipur: A cross-sectional study. Indian J Public Health. 2020;64(2):173-7.

23. Giri BR, Namgyal P, Tshering K, Sharma K, Dorji T, Tamang C. Mass measles rubella immunization campaign: bhutan experience. Indian J Community Med. 2011;36(2):109-13.

\section{Figures}

\section{Figure 1}

Distribution of AEFI cases during MR catch-up campaign: October 2019 


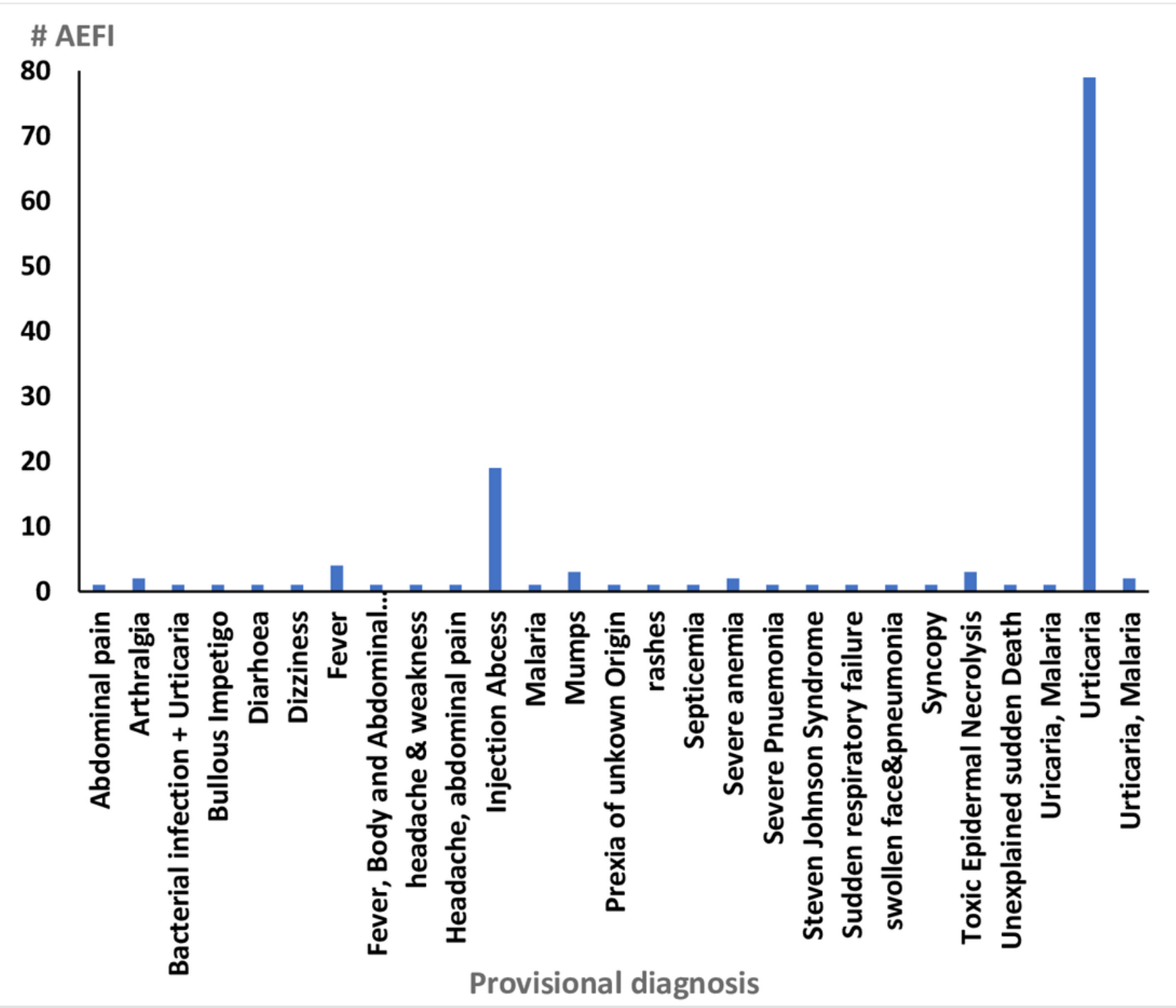

Figure 2

Provisional diagnoses of the different AEFI during the Oct 2019 MR catch-up campaign 